\title{
Motion Recognition for Stroke Rehabilitation Based on BP, RBF Neural Network and Support Vector Machine
}

\author{
Li-Quan Guo, Ji-Ping Wang, Da-Xi Xiong \\ Jiangsu Key Laboratory of Medical Optics \\ Suzhou Institute of Biomedical Engineering and \\ Technology, Chinese Academy of Sciences \\ Suzhou, China \\ E-mail: guolq@sibet.ac.cn
}

\author{
Jie-Yong Bian, Lin-Qiang Zhou \\ Department of Neurology, \\ Suzhou Xiangcheng People's Hospital, \\ Suzhou, China \\ E-mail:18662204700@163.com
}

\begin{abstract}
In order to monitor the rehabilitation training of stroke patients in unsupervised situation and provide rehabilitation advice for rehabilitation clinicians, a wireless upper limb motion recognition system has been developed using 9-axis sensors, to identify the complex upper limb movements from stroke patients' rehabilitation program, such as Bobath handshake, paraplegia hand touch shoulder, elbow flexion and extension, shoulder joint horizontal outreach and elbow flexion touch head. 155 different exercises from 9 stroke patients' rehabilitation training program were adopted to verify and validate the system with 100 of them in the training group and the other 55 in the testing group. After preprocessing and the feature extraction of the acquired motion data of the data of training group, BP Neural Network, Radial Basis Function (RBF) Neural Network and Support Vector Machine (SVM) recognition approach were employed to establish three small sample identification models. Then, the data of testing group in the upper limb rehabilitation training program were used to identify the developed models. Finally, the results of three kinds of motion recognition models were compared and analyzed. It had been found that the recognition accuracy of the developed models was above $90 \%$ respectively, and SVM model had less time consuming and higher accuracy. This result provides a well reference for further development of an automated system for stroke patient rehabilitation motion recognition.
\end{abstract}

Keywords-motion recognition; $B P$ neural network; $R B F$ neural network; support vector machine

\section{INTRODUCTION}

Nowadays, with the improvement of people's living standard and the rapid aging of population in many countries, stroke has become a leading cause of death and ongoing disability in the world. According to an incomplete statistics, there are about 1.5 million patients died from stroke each year in China, and there are more than 2 million new stroke patients each year [1]. On the other hand, in most developing countries, there is no enough rehabilitation centers or other rehabilitation institutions for treating stroke patients. At the same time, the healthcare expenditure dedicated to stroke related diseases is becoming higher and higher which is out of the affordability of many families. Therefore, many current researches have been focused on developing unsupervised rehabilitation methods which enable the patients to perform scheduled rehabilitation training outside medical facilities. For stroke patients, one major problem to be solved in this research is to find a reliable continuous monitoring method that can provide real-time feedback of patient's training progress to the responsible medical professionals and at the same time ensure the patient's safety [2].

During the rehabilitation process, a patient's motion is one of the most difficult tasks to be recorded. At present, the common human motion-capturing methods are visual based tracking systems which utilize optical sensors and visual markers to track the body movements. Visual based tracking systems provide higher standard accuracy in general. However, the systems using visual markers may experience occlusion problem which means these systems will have a problem capturing the movements that involves body overlapping and joint rotating while these movements can commonly be found in rehabilitation training exercises $[3,4]$. Visual based systems are also relatively heavy, costly, and difficult to set up or calibrate which make it not suitable for home-based applications[5].

According to that, this paper presents a wireless motion tracking system, including two 9-axis sensors and a receiver module. Respectively, the 9-axis sensors are fixed in forearm and upper arm affected, using to collect and record the rehabilitation training data. Due to its small size, low power consumption and low cost, the system is very suitable for the application of unsupervised home rehabilitation monitoring.

\section{SYSTEM DESCRIPTION}

During the rehabilitation training progress, the training data is collected by two 9-axis sensors including 3-axis accelerometer,3-axis gyroscope and 3-axis magnetometer, sent to the receive module wirelessly, and then sent to a PC via USB interface. On the PC, the raw data is stored on the hard disk and displayed on the screen. The patients can then watch their real time training motions. After preprocessing, the training data is then sent to the motion recognition classifier to identify which actual movement the patient just did. The identified result is sent to the server in hospital via internet for clinician's perusal. The rehabilitation specialists can then view and analyze the patients' training quality and the current impairment condition from the uploaded patients' body and limb movement data on the server, and provide advice and decision on patient's next training session. The system architecture is illustrated in Figure 1[6]. 


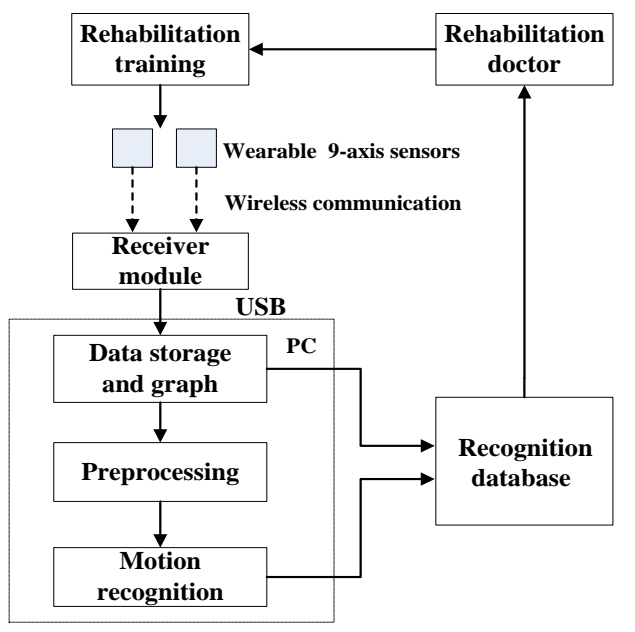

Figure 1. The scheme of hand motion tracking system.

\section{EXPERIMENTS}

\section{A. Data Sampling}

In order to examine the performance of the proposed system, an experiment involved in 9 stroke patients was carried out, approved by Suzhou Xiangcheng People's Hospital Ethics Committee. Compared to other rehabilitation exercises, the upper limb rehabilitation exercises are more complex and more representative. Under the rehabilitation doctor's advice, several typical rehabilitation movements, such as Bobath handshake, paraplegia hand touch shoulder, elbow flexion and extension, shoulder joint horizontal outreach and elbow flexion touch head were used in the experiment. The patients were asked to repetitively perform these common upper limb exercises with wireless inertia sensors attached to their arms, as shown in Figure 2.

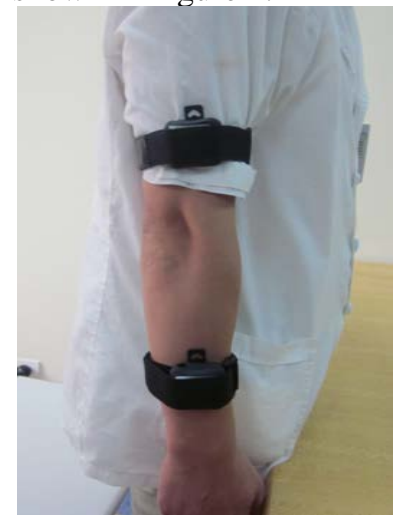

Figure 2. Fixed position of the sensors.

The exercises were sampled under relatively loose supervision, which means the movements recorded were performed at various speed and completeness, in order to simulate the real practice situation.

\section{B. Data Preprocessing and Feature Extraction}

For each movement, the patients were asked to repeat 5 times, each time as an exercise, according to the rehabilitation clinician's suggestion. The motion tracking system operated in a period of 30 to 50 seconds, according to the physical condition of the patients.

During the whole data acquisition process, all the training exercises of the patients were guided by a rehabilitation doctor. Therefore, the raw data was gathered according to the real practice situations.

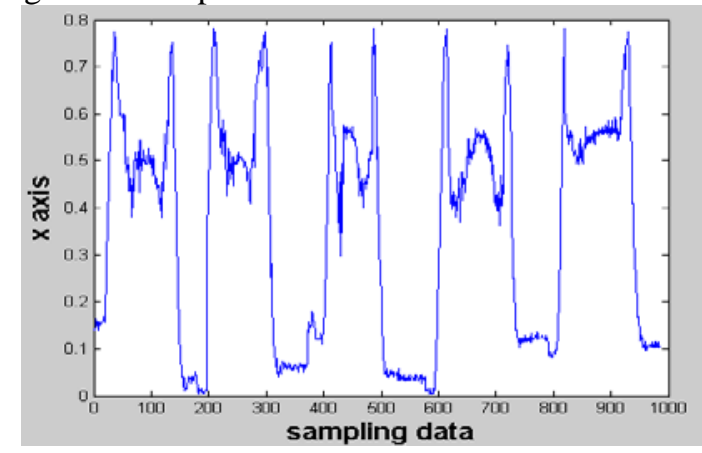

Figure 3. The raw data gathered from a patient doing paraplegia hand touch shoulder.

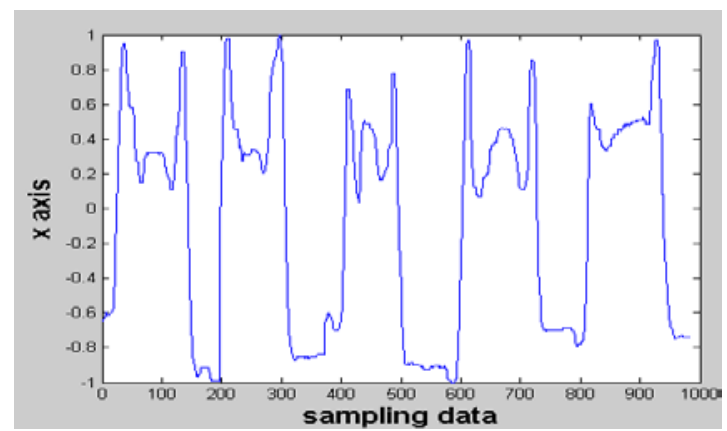

Figure 4. The denoised data using wavelet transform denoising method.

Figure 3 shows the upper limb movement data gathered from one of the 9-axis tilt sensors while a patient doing the paraplegia hand touch shoulder motion. It is obvious that the raw signal has 5 movements, but also contains noise caused by the patient's random shaking and other irrelevant motion components. By using wavelet transform decomposition and then thresholding the detail coefficients of each scale, the noise in raw signal can be suppressed nicely. Figure 4 shows the denoised data from which it can be seen that the signal is smoother after preprocessing.

Considering the number of sampling points in each movement is about 110 , we can't take all the sampling points as the input attributes of the motion recognition model, therefore it is quite necessary to extract some features as the input attributes. After preliminary study ant test of the experimental sample, total 12 attributes, 6 attributes from each tilt sensor were extracted and used. They are the initial position and accelerometer amplitude of $\mathrm{x}, \mathrm{y}$ and $\mathrm{z}$. 


\section{MODELING AND RESULT ANALYSIS}

\section{A. Training Samples and Test Samples}

Due to differences of the patient's rehabilitation, some patients with severe condition could not complete all the rehabilitation training movements, 155 effective exercises were collected finally. For each movement, 20 exercises randomly selected, there were 5 movements total 100 exercises as the training group samples. The other 55 exercises were in the test group, including 15 Bobath handshake, 15 paraplegia hand touch shoulder, 15 elbow flexion and extension, 5 shoulder joint horizontal outreach and 5 elbow flexion touch head. In order to ensure the accuracy and objectivity, the data of training group and the test group were denoised and extracted features as the same. After that, BP Neural Network, RBF Neural Network and SVM were established based on the training group data.

\section{B. BP Neural Network and Results}

BP Neural Network, one of the most popular techniques in the field of Neural Network, is a kind of supervised learning neural network, the principle behind which involves using the steepest gradient descent method to reach any small approximation. There are three layers contained in BP: input layer, hidden layer, and output layer. Two nodes of each adjacent layer are directly connected, which is called a link. Each link has a weighted value presenting the relational degree between two nodes.

The motion recognition results of $\mathrm{BP}$ model on the testing group are shown in Figure 5, where in $\mathrm{x}$-axis represents the number of samples, $y$-axis shows a sample type. In the 55 exercises, there are two recognition mistakes in Bobath handshake, one recognition mistake in paraplegia hand touch shoulder and one recognition mistake in elbow flexion and extension.

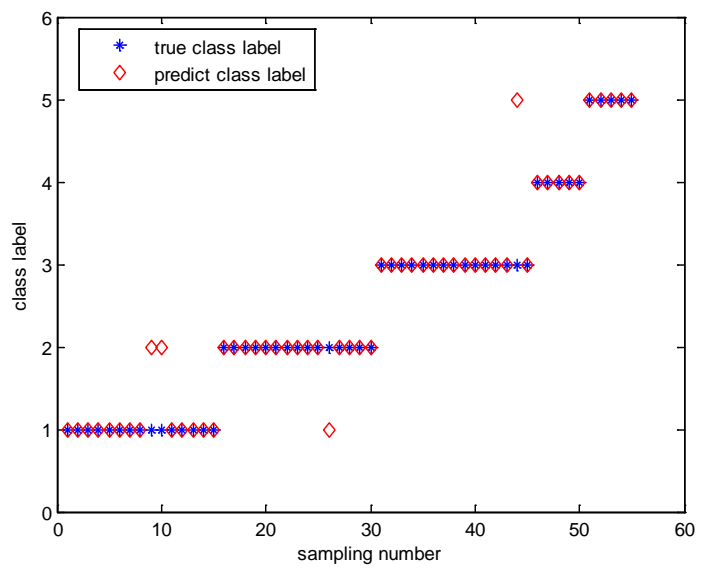

Figure 5. Motion recognition results of BP model.

\section{RBF Neural Network and Results}

RBF Neural Network is a good performance threeforward neural network, with the nature of a global approach, but no local optimization problem, as while as advantages such as compact topology, configuration parameters learning discretely fast convergence, etc. Typically there are three layers: an input layer, a hidden layer with a non-linear RBF activation function and a linear output layer.

The motion recognition results of RBF model on the testing group are shown in Figure 6, where in $\mathrm{x}$-axis represents the number of samples, y-axis shows a sample type. In the 55 exercises, there are four recognition mistakes in paraplegia hand touch shoulder and one recognition mistake in elbow flexion and extension.

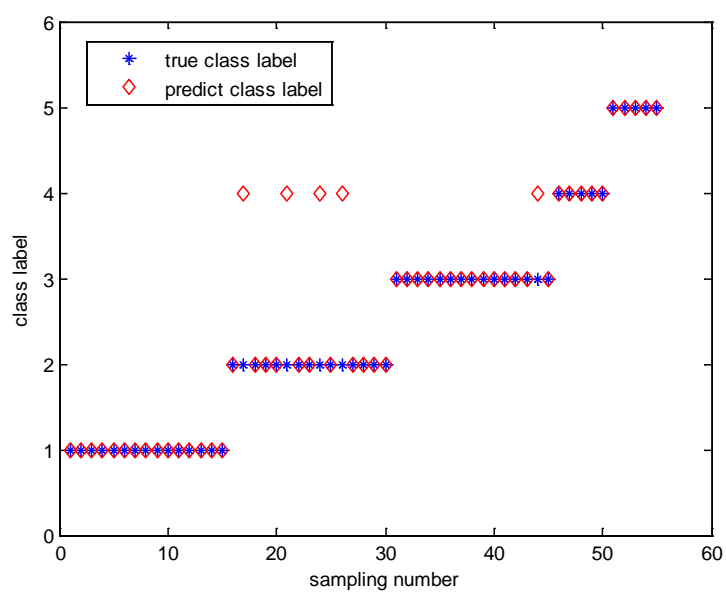

Figure 6. Motion recognition results of RBF model.

\section{SVM and Results}

Compared with other traditional supervised and unsupervised neural network based techniques, SVM can achieve better generalization with a small set of training samples. The following is a basic introduction of SVM [7].

Given training vectors $x_{i} \in R^{n}, i=1, \ldots, l$, in two classes, and a vector $y \in R^{l}$ such that $y_{i} \in\{1,-1\}$. The goal is to find a hyperplane which can classify these two classes correctly.

Suppose the hyperplane is described by

$$
\mathbf{w} \cdot \mathbf{x}+b=0
$$

Consequently, the above classification problem can be write as

$$
\left\{\begin{array}{l}
\mathbf{w} \cdot \mathbf{x}_{i}+b \geq 1, \quad y_{i}=1 \\
\mathbf{w} \cdot \mathbf{x}_{i}+b \leq-1, \quad y_{i}=-1
\end{array} \quad i=1, \ldots, l\right.
$$

Theoretically, there may exist infinite hyperplanes satisfy (2), the task of SVM is to find the best hyperplane which is the farthest one from the training set[8].

The motion recognition results of SVM model on the testing group are shown in Figure 7, where in $\mathrm{x}$-axis represents the number of samples, $y$-axis shows a sample type. In the 55 exercises, there are two recognition mistakes 
in paraplegia hand touch shoulder.

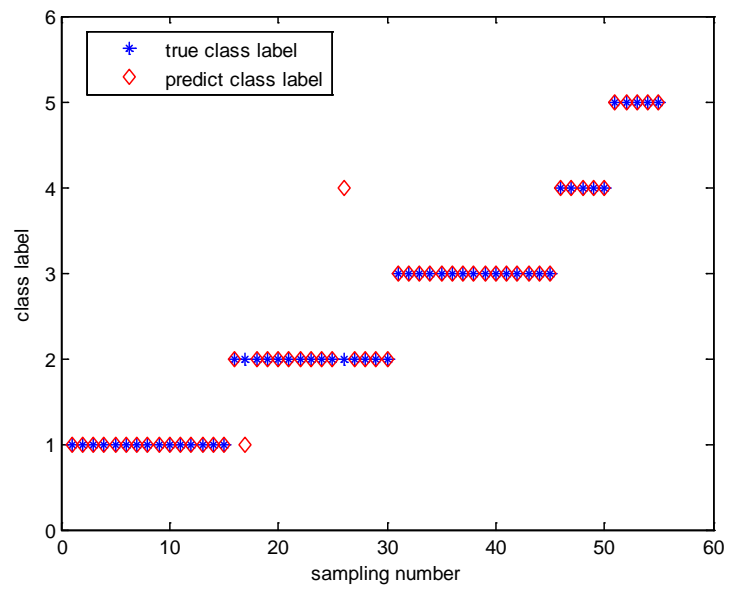

Figure 7. Motion recognition results of SVM model.

\section{RESULTS AND DISCUSSION}

\section{A. Comparison Results}

After the models trained by solving the above optimization problems, users can predict labels of testing data. Generally, we can evaluate the predictions by the following measures.

$$
\text { Accuracy }=\frac{\# \text { correctly predicted data }}{\# \text { total data }} \times 100 \%
$$

As while as the accuracy, training time of the three models and the testing time of the test group was also been calculated and compared. Because each training time and testing time of the network was different, 1000 program execution time were averaged. The results are shown as in TABLE I. It can be seen that the SVM model has the best recognition accuracy and least training time obviously, which is most suitable for the wireless upper limb motion recognition system.

TABLE I ACCURACY AND PROGRAM EXECUTION TIME OF BP, RBF AND SVM

\begin{tabular}{|c|c|c|c|}
\hline Time & BP & RBF & SVM \\
\hline Training & 0.3894 & 0.0779 & 0.0121 \\
\hline Simulation & 0.0136 & 0.0113 & 0.0113 \\
\hline execution & 0.3395 & 0.3395 & 0.3395 \\
\hline Total & 5.105 & 5.5258 & 4.015 \\
\hline Accuracy & $90.91 \%$ & $90.91 \%$ & $96.36 \%$ \\
\hline
\end{tabular}

\section{B. Discussion and the Future Work}

The experiment results indicate that the established models based on BP, RBF and SVN can recognize and classify the rehabilitation training motion types such as Bobath handshake, paraplegia hand touch shoulder, elbow flexion and extension, shoulder joint horizontal outreach and elbow flexion touch head, and SVM has the best recognition accuracy and least training time.

However, the experiment cannot instead the real status of rehabilitation motions wholly, so it is necessary to collect more patients' data for further experiment. Additionally, it's necessary to find better feature extraction method to fully represent the status of the real motion and establish an online recognition model.

\section{CONCLUSION}

In order to monitor the hand rehabilitation training of stroke patients in unsupervised or remote situation, a wireless measurement tracking system for hand motion recognition has been developed using 9-axis sensors, with motion recognition models based on BP, RBF and SVM. The experiment results indicate that all the models can recognize the five typical and common upper limb movements Bobath handshake, paraplegia hand touch shoulder, elbow flexion and extension, shoulder joint horizontal outreach and elbow flexion touch head respectively, with the accuracy all above $90 \%$. As while as the accuracy, the training time of the three models and the testing time of the test group was also been calculated and compared. The results indicate that SVM model has the best recognition accuracy and least training time obviously, which provides a well reference for further development of a fully automated and real-time system for stroke patient rehabilitation motion recognition.

\section{ACKNOWLEDGMENT}

Thanks given to Doctor Jie-yong Bian and Doctor Linqiang Zhou from Suzhou Xiangcheng People's Hospital, Jiangsu Province, China, for their great assistance during the data collection process. The clinical experiment involved human participants were approved by the Ethics Committee of Suzhou Xiangcheng People's Hospital.

\section{REFERENCES}

[1] M. L. Rao, "China Guideline for Cerebrovascular Disease Prevention and Treatment,” People's Medical Publishing House, Beijing, 2007, ch. 1.

[2] H. M. Dalal, P. H. Evans, J. L. Campbell et al. "Home-based Versus Hospital-based Rehabilitation after Myocardial Infarction: A Randomized Trial with Preference Arms - Cornwall Heart Attack Rehabilitation Management Stud," International Journal of Cardiology, 2007, pp. 119: 202-211.

[3] H. Y. Zhou, and H. S. Hu. "Inertial motion tracking of human arm movements in stroke rehabilitation," International Conference on Mechatronics \& Automation, Niagara Falls, Canada, 29 July- 1 August, 2005,1306-1311.

[4] Zhe Zhang, Qiang Fang, Ferry, Ferry, "Upper limb motion capturing and classification for unsupervised stroke rehabilitation," IECON 2011-37th Annual Conference on IEEE Industrial Electronics Society, vol., pp. 3832-3836, 7-10 Nov. 2011.

[5] Zhi-Qiang Zhang, Jian-Kang Wu et al, "A Novel Hierarchical Information Fusion Method for Three-Dimensional Upper Limb Motion Estimation.” IEEE Transactions on instrumenttation and measurement, vol. 60, NO. 11, Nov 2011.

[6] Liquan Guo, Lei Yu, Qiang Fang et al. ”Upper Limb Motion Recognition for Unsupervised Stroke Rehabilitation Based on Support Vector Machine," ISBB International Symposium on Bioelectronics and Bioinformatics, 3-5 Nov. 2011, pp. 37-40. 
[7] C. W. Hsu, and C. J. Lin. "A comparison of methods for multi-class support vector machines," IEEE Transactions on Neural Network, 2002, vol. 13, no. 2, pp. 415-425.

[8] Liquan Guo, Jiping Wang, Qiang Fang et al.” Motion recognition for unsupervised hand rehabilitation using support vector machine," Biomedical Circuits and Systems Conference (BioCAS), 28-30 Nov. 2012. 